\title{
Effects of Seasonal Climatic Conditions on the Diagnosis of Mycobacterium avium Subspecies paratuberculosis in Dairy Cattle*
}

\author{
S. J. Strickland, ${ }^{1}$ H. M. Scott, ${ }^{1}$ M. C. Libal, ${ }^{2} \dagger$ A. J. Roussel Jr., ${ }^{3}$ and E. R. Jordan ${ }^{4}$ \\ ${ }^{1}$ Department of Veterinary Integrative Biosciences, \\ ${ }^{2}$ Texas Veterinary Medical Diagnostic Laboratory, \\ ${ }^{3}$ Department of Veterinary Large Animal Clinical Sciences, and \\ ${ }^{4}$ Department of Animal Science, Texas A\&M University, College Station 77843
}

\section{ABSTRACT}

Validity of Johne's disease programs and control protocols that rely on established cut points [e.g., specified sample-to-positive (S/P) ratios] for ELISA serological tests depends on interpreted results that are not susceptible to variable test accuracy. It was hypothesized that seasonal variability exists in serological response to Mycobacterium avium subsp. paratuberculosis (MAP) infection. Further, a reciprocal response may occur, resulting in greater risk of fecal shedding in subclinically infected animals. A testing regimen was invoked that included multiple testing of individual adult cows during the 4 seasons. Serum was collected on a cyclic, monthly basis from 3 randomly selected cohorts of dairy cows, and fecal samples were collected from the $20 \%$ of cows with the greatest ELISA test S/P ratios. Staggered, quarterly sampling was continued for $1 \mathrm{yr}$, and at the conclusion, serum was analyzed en masse. The ELISA outcome values (i.e., S/P ratio) were treated both as categorical and continuous variables. The potential lagged effects of temperature-related seasonality on S/P ratio, as well as the potential for a change in test result caused by temperature were assessed. Results for fecal culture were analyzed on a categorical scale and compared with the ELISA results to explore the possibility of reciprocal fecal shedding. No significant seasonal effects on either $\mathrm{S} / \mathrm{P}$ ratios or the proportion of cows seropositive to MAP were observed. Furthermore, no evidence was found linking temperaturerelated seasonality to a reciprocal increase in the risk of fecal culture positivity for MAP.

Received October 9, 2004.

Accepted March 31, 2005.

Corresponding author: H. M. Scott; e-mail: HMScott@cvm. tamu.edu.

*Supported in part by USDA-APHIS Grant No. 02-9148-0717-GR. Any opinions, findings, conclusions, or recommendations expressed in this publication are those of the authors and do not necessarily reflect the view of the U.S. Department of Agriculture.

$\dagger$ Present affiliation: Department of Veterinary Pathobiology.
(Key words: Johne's disease, Mycobacterium avium subsp. paratuberculosis, seasonality, temperature)

Abbreviation key: AR = auto-regressive, $\mathbf{J D}=$ Johne's disease, GEE = generalized estimating equation, $\mathbf{M A P}=$ Mycobacterium avium subsp. paratubercu losis, $\mathbf{S} / \mathbf{P}=$ sample-to-positive, $\mathbf{T H I}$ = temperature-humidity index, TVMDL = Texas Veterinary Medical Diagnostic Laboratory.

\section{INTRODUCTION}

Johne's disease (JD) is a chronic, enteric disease of ruminants caused by infection with Mycobacterium avium subsp. paratuberculosis (MAP), otherwise known as Mycobacterium paratuberculosis. This disease is endemic within the United States, with at least $22 \%$ of dairy herds classified as Johne's positive (NAHMS, 1997). Production, reproduction, and culling losses caused by JD can be significant (Goodell et al., 2000). Potential economic losses attributable to this disease are substantial.

No effective, practical treatment for JD exists; however, it can be controlled through implementation of strategies that are at least partially dependent on the identification and management of MAP-infected individuals. On-farm biosecurity, such as feeding pasteurized milk or milk replacer to neonatal calves, increasing farm sanitation to minimize fecal-oral transfer of MAP, and the purchasing of animals from known low-risk sources, are also thought to be important in controlling spread of the disease. To best implement appropriate measures, however, producers must first be aware of the extent of the presence of JD in their herds. In an effort to improve awareness and minimize the spread of JD, the US Animal Health Association, (USAHA, 1998) in conjunction with federal and state agencies, initiated a Voluntary Bovine JD Status Program for cattle (USDA-APHIS, 2002). One of the program's objectives is to identify low-risk herds to minimize the inter-herd spread of disease through outside purchase of replacement animals. Programs such as the Volun- 
tary Bovine JD Status Program utilize standardized diagnostic tests to assess infection status; thereby providing increased confidence for freedom from disease at the herd level, provided that all animals test negative during strategically designed testing schemes.

Detecting subclinical animals (i.e., infected but not exhibiting clinical signs) is considered by many to be extremely important to the control of the spread of disease. Unfortunately, the pathogenesis of JD complicates the application and interpretation of these tests (e.g., fecal culture and/or serological testing). Test specificity is usually high, but a low sensitivity makes it very difficult to detect all animals in a herd that are infected (Dargatz et al., 2001; Nielsen et al., 2002). Because the role of these tests is crucial to JD control programs, potential problems affecting sensitivity and specificity also need to be identified and well characterized.

\section{Background}

In early 2001, during the conduct of a herd-health program designed to minimize calfhood exposure to MAP on a dairy farm in Texas, a seasonal pattern of serological response to MAP was observed. Roughly once per month, dairy cows that were confirmed pregnant were tested for serum antibodies to MAP using a commercial ELISA test. Upon preliminary examination of those data, a decrease in the proportion of animals testing positive for JD during summer months was noted (May through August). This decrease is illustrated in a categorical scale (i.e., proportion testing positive) in Figure 1. Previous studies have shown that cattle experiencing hyperthermia (i.e., heat stress) have reduced productivity and feed intake, as well as altered endocrine function and energy balance (Wolfenson et al., 2000; De Rensis and Scaramuzzi, 2003), so it is biologically plausible that elevated temperatures also will have an effect on cattle immune response to MAP infection. Indeed, although varied and conflicting results exist in the literature concerning the relationship between heat stress (climate-related seasonality) and immunological traits such as serum and colostral immunoglobulin concentrations (Lacetera et al., 2002), the degree of heat stress experienced by a dairy cow seems to play an important role in the response to infection.

Based on our preliminary cross-sectional data, the primary objective of this project was to examine seasonal temperature effect on the interpreted results of serological testing using a commercial ELISA for MAP in a large Texas dairy cattle herd. An epidemiological field study design was employed to control for the effects of age (i.e., birth cohort) and other potential confounders on the interpretation of a seasonal decrease in the proportion or risk of adult cows being categorized as

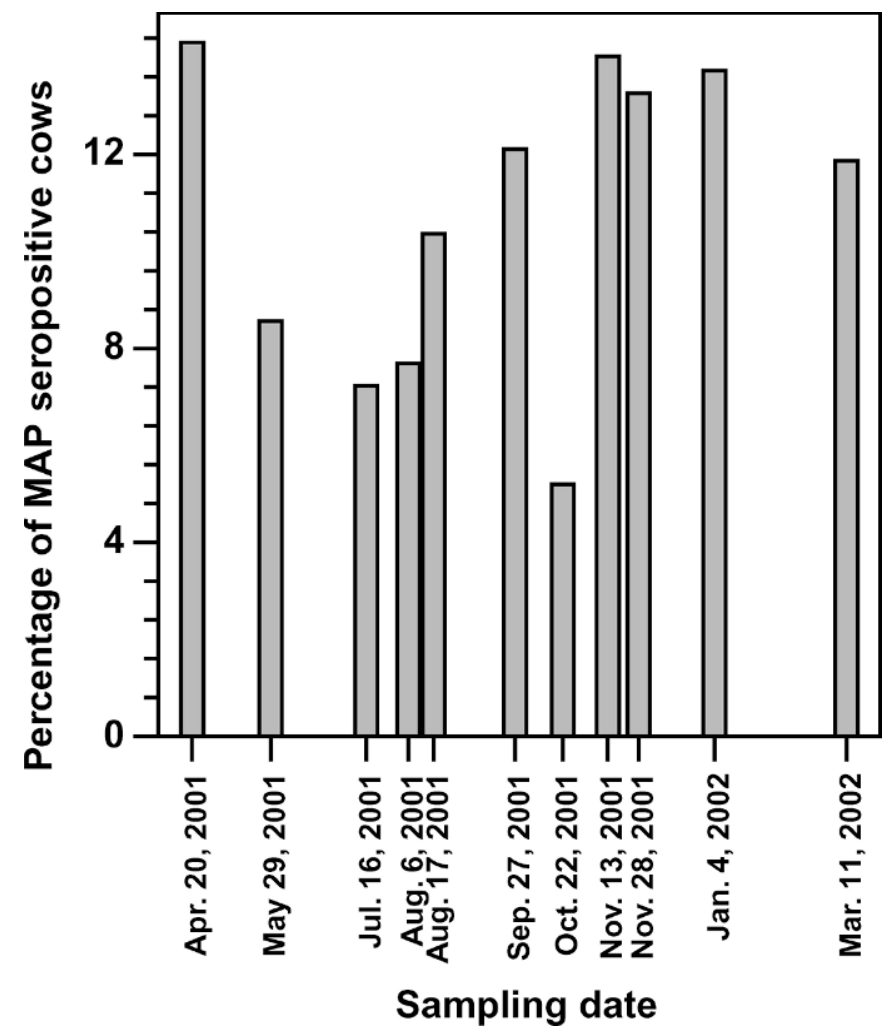

Figure 1. Historical cross-sectional time-series preliminary data illustrating the percentage of cows testing seropositive each month for Mycobacterium avium subsp. paratuberculosis (MAP) using the ELISA kit manufacturer's established sample-to-positive (S/P) ratio cut point of $\geq 0.25$.

positive for JD under a contemporary cohort sampling scheme. In addition, the potential for a change in test result (negative to positive or vice versa) for the same cow was examined with a repeated seasonal sampling schedule.

A secondary objective of this project was exploration of the possibility of a reciprocal increase in the risk of fecal shedding for those periods during which seasonal changes related to heat might reduce humoral response as measured by the sample-to-positive (S/P) ratio of the commercial ELISA.

\section{MATERIALS AND METHODS}

\section{Study Population}

The cooperator herd consisted of approximately 3400 lactating and dry cows located on a commercial dairy in Texas. Cows were milked $3 \times / \mathrm{d}$ in a double-50 parallel parlor, with a rolling herd average $>11,000 \mathrm{~kg}$ of milk. Because of expansion of the herd, just over one-half of the cows had been purchased from outside sources. Approximately $69 \%$ of the cows were in their first lacta- 
Table 1. Number of cattle sampled at first season (cohorts 1, 2, and 3) and lost to follow up.

\begin{tabular}{llcc}
\hline Cohort & $\begin{array}{l}\text { Sampled at } \\
\text { first visit }\end{array}$ & Culled & Death \\
\hline & & $($ no. $)$ & \\
\cline { 2 - 4 } 1 & 226 & 55 & 24 \\
2 & 223 & 41 & 23 \\
3 & 210 & 40 & 12 \\
\hline
\end{tabular}

tion, $16 \%$ were in their second lactation, and $15 \%$ were in their third or greater lactation, with an annual culling rate of 35 to $40 \%$. Herd production and health events were recorded and tracked using a commercial on-farm computer software program (DairyComp 305; Valley Agricultural Software, Tulare, CA). All cows were housed in areas with access to shade; however, soakers and fans were not used. Although the farm had implemented a program to minimize MAP infection by managing colostrum based on ELISA test results from dams, the owner indicated that cows were not culled based on test results.

\section{Sampling Scheme}

Eligible cows were identified through the farm's computerized records, excluding only those from the sampling frame that were both not pregnant and late in their lactation, which increased the likelihood of culling early in the study. Cows to be randomly sampled were stratified on the basis of DIM and lactation number; then, selection was weighted toward detecting those at greatest risk for identification of JD with $50 \%$ of those selected in their third or greater lactation, $35 \%$ in their second lactation, and $15 \%$ in their first lactation. Based on the historically observed differences between the proportion testing positive using the ELISA during summer months (approximately 8\%) vs. winter months (approximately 13\%), with 95\% confidence and 80\% power, 463 cows were required (one-sided test). Because we expected some attrition over a full year, resulting from normal herd culling procedures, we sampled more than were needed. Therefore, cows were randomly allocated into 3 separate monthly sampling groups of approximately 220 head each, which were resampled in staggered periods separated by 3-mo intervals, resulting in 4 sample collection periods per group. In the end, a total of 539 cows actually had repeated samples collected during the study period. Numbers of cows sampled initially (by cohort) and numbers lost to culling or death are presented in Table 1.

\section{Sampling Procedure}

At the start of the study (month 1, October 2002), approximately $10 \mathrm{~mL}$ of blood were collected from each cow in the first group of cows. The sera from these blood samples were evaluated for antibodies to JD using a commercial ELISA test. For this first sampling period only, the $20 \%$ of cows with the highest S/P ratio, as reported by the laboratory, were then resampled shortly after the initial blood collection. At least $25 \mathrm{~g}$ of feces were collected from each cow and submitted to the laboratory for subsequent fecal culture. Fecal samples were collected directly from the rectum in sterile palpation sleeves using deep-well water from the milk house tap as the only lubricant. The same cows designated as the top $20 \%$ by S/P ratio [calculated using the formula provided by the manufacturer: (mean optical density (OD) of sample - OD of negative control)/(OD of positive control - OD of negative control); IDEXX Laboratories Inc., 2002] at the first testing period were re-sampled for fecal culture at each of the subsequent 3 seasonal sampling periods. This process was repeated for each group during the first $3 \mathrm{mo}$, then the cycle was repeated for the remaining 3 seasons, beginning with the first group of cows. The producer was blinded to the ELISA and fecal culture sample results of cattle during the study period.

\section{Laboratory Procedure}

Serum antibody. After collection, all blood samples were taken to the Texas Veterinary Medical Diagnostic Laboratory (TVMDL) and centrifuged. Serum was decanted into serum tubes. A commercial solid-phase ELISA kit (HerdChek; IDEXX Laboratories Inc.,Westbrook, ME) was used to analyze serum from the blood samples collected. All experimental samples were analyzed in duplicate wells per laboratory protocol following the manufacturer's instructions. After initial evaluation of the first 3 groups to determine the $20 \%$ of cows with highest $\mathrm{S} / \mathrm{P}$ ratios, all samples (including those collected during the first season) were subsequently maintained frozen at $-70^{\circ} \mathrm{C}$ upon arrival at TVMDL. At the completion of the collection period of the study, all serum samples were thawed and evaluated in duplicate per TVMDL protocol. To limit plate-to-plate and inter-operator variation, each set of samples collected per cow (up to 4) was analyzed on the same plate using a Biomek FX robotic device (Beckman Coulter, Fullerton, CA). Samples were allocated randomly on the plate to minimize potential bias caused by within-plate variation.

Fecal culture. Upon arrival at TVMDL, all fecal samples were frozen and maintained at $-70^{\circ} \mathrm{C}$. The fecal culture procedure generally followed that of Stabel (1997). Throughout the study period, each sample was thawed and 2 to $3 \mathrm{~g}$ of feces were placed in a $50-\mathrm{mL}$ centrifuge tube and filled to $35 \mathrm{~mL}$ volume with distilled 
water. Samples were mixed on a rotating mixer for 30 min. Samples were left standing for $30 \mathrm{~min}$, and the supernatant fraction was decanted into a new $50-\mathrm{mL}$ tube and centrifuged at $1700 \times g$ for $20 \mathrm{~min}$. Supernatant was discarded, and the pellet was resuspended in $30 \mathrm{~mL}$ of $0.9 \%$ cetylpyridinum chloride $/ 1.9 \%$ brain heart infusion. Samples were then incubated overnight at $37^{\circ} \mathrm{C}$ as a decontamination step and then centrifuged at $1700 \times g$ for $20 \mathrm{~min}$. Supernatant was discarded, and the pellet was resuspended in $1 \mathrm{~mL}$ of sterile water with $50 \mu \mathrm{g} / \mathrm{mL}$ amphotericin $\mathrm{B}, 100 \mu \mathrm{g} / \mathrm{mL}$ vancomycin, and $100 \mu \mathrm{g} / \mathrm{mL}$ nalidixic acid. Samples were again incubated overnight at $37^{\circ} \mathrm{C}$ and then inoculated onto Herrold's egg yolk medium $(0.2 \mathrm{~mL}$ per tube $)$ with 4 tubes containing Mycobactin $J$ and one tube without. Tubes were placed in a slanted position with caps loosened and incubated at $37^{\circ} \mathrm{C}$. Samples were checked for contamination, caps were tightened, and tubes placed in an upright position after $1 \mathrm{wk}$. Samples were then incubated for up to $15 \mathrm{wk}$ and checked weekly for appearance of MAP. If no growth was visible after $15 \mathrm{wk}$, samples were determined to be negative. If colonies typical for MAP were observed at 15 wk (i.e., those growing on Mycobactin J only), they were stained using cold acid-fast stain. Acid-fast colonies were confirmed positive for MAP by PCR, a test used to detect IS900, a genomic insertion sequence specific for MAP using a protocol modified from Khare et al. (2004).

\section{Climatic Data}

Climatic conditions were assessed using data from a local weather station, including monthly mean, monthly mean minimum, and monthly mean maximum temperatures (mean minimum and mean maximum refer to daily measurements). An on-farm weather station was erected during the study; however, because data were not available for the entire study, the highly correlated data $\left(R^{2}=0.997\right)$ from the local weather station (distance $=15 \mathrm{~km})$ was used instead.

\section{Statistical Analyses}

Variables assessed in the analyses included the following: seropositivity of cows to MAP antigen (binary classification via ELISA); S/P ratio results (interval variable from ELISA); fecal culture result (binary classification); age (based on lactation number); stage of lactation (DIM); and mean, mean daily minimum, and mean daily maximum monthly temperatures. Lactation was evaluated as a 4-level categorical variable to avoid statistical model instability, attributable to insufficient numbers in the highest lactations (i.e., 5 to 8). Categories 1 to 3 corresponded with lactations 1 to 3 ; category
4 included lactations 4 to 8 . Days in milk also were evaluated as a categorical variable representing physiologically important stages of lactation. Category 1 included 0 to 30 DIM, category 2 included 31 to 60 DIM, category 3 included 61 to 150 DIM, category 4 included 151 to 305 DIM, and category 5 included >305 DIM.

Effects of various current and lagged temperaturerelated seasonal climatic factors on the risk of cows exhibiting seropositivity were tested in a generalized linear modeling framework (PROC GENMOD; SAS Inst., Inc., Cary, NC) using a binomial distribution and a logit link function. Using the repeated statement (by cow) and an auto-regressive [AR(1)] correlation structure, a generalized estimating equation (GEE) was utilized to adjust for the within-cow dependence of the outcome variable over repeated sampling (Diggle et al., 2002). Potential explanatory variables that were analyzed as categorical variables included sampling cohort; season of sampling; sampling month; fecal culture result; lactation number (categorized); and mean, mean daily minimum, and mean daily maximum temperatures for the month of, month before, and 2 mo before sampling. Effect of climatic factors on actual continuous S/P ratio was assessed using a mixed modeling framework (PROC MIXED; SAS Inst., Inc.) with random effects for cow (with an AR(1) correlation structure) and fixed effects for climate and cow-level factors such as lactation number. The $\mathrm{S} / \mathrm{P}$ ratio was not log-transformed because negative $\mathrm{S} / \mathrm{P}$ values were possible. This model utilized the same variables as the GLM framework.

Effect of climatic factors on $\mathrm{S} / \mathrm{P}$ ratio (categorized into quintiles) was assessed in a generalized linear modeling framework using a multinomial distribution and a cumulative logit link function (McCullagh and Nelder, 1989; Hardin and Hilbe, 2003). Using the repeated statement (by cow) and an independent correlation structure, a GEE was utilized to adjust for the withincow dependence of the outcome variable. An AR correlation structure would have been ideal, but the software procedure would not permit a correlation structure other than independent (Hardin and Hilbe, 2003) for the cumulative logit model. However, the GEE parameter estimates remain robust even if correlation structure is misstated (SAS, 1996). The GEE parameter estimates are based on robust estimates of the standard errors derived from the empirical covariance matrix.

A transitional model based on a first-order Markovchain for binary data (Diggle et al., 2002) was used to model the dependence of change or no change in positive-negative status and was based on the same explanatory variables as described previously. A cow was considered to be positive if analysis of serum antibodies by ELISA resulted in an $\mathrm{S} / \mathrm{P}$ ratio $\geq 0.25$ (IDEXX Labora- 
Table 2. ELISA $\left[\mathrm{S} / \mathrm{P}^{1}\right.$ ratio and positive (+) or negative (-)] and fecal culture (FC) results (+/-) for each season by cohort.

\begin{tabular}{lllclcl}
\hline $\begin{array}{l}\text { Season } \\
\text { by cohort }\end{array}$ & $\begin{array}{l}\text { Mean S/P } \\
(\mathrm{SE})\end{array}$ & $\begin{array}{l}\text { Median } \\
\mathrm{S} / \mathrm{P}\end{array}$ & $\begin{array}{l}\text { ELISA } \\
+\end{array}$ & $\begin{array}{l}\text { ELISA } \\
-\end{array}$ & $\begin{array}{l}\text { FC } \\
+\end{array}$ & $\begin{array}{l}\text { FC } \\
-\end{array}$ \\
\hline Fall & $0.076(0.011)$ & 0.023 & 24 & 425 & 8 & 82 \\
1 & $0.042(0.009)$ & 0.027 & 4 & 152 & 2 & 36 \\
2 & $0.094(0.021)$ & 0.022 & 12 & 152 & 3 & 26 \\
3 & $0.094(0.024)$ & 0.023 & 8 & 121 & 3 & 28 \\
Winter & $0.097(0.014)$ & 0.023 & 34 & 418 & 6 & 67 \\
1 & $0.076(0.023)$ & 0.024 & 8 & 144 & 1 & 22 \\
2 & $0.113(0.027)$ & 0.020 & 13 & 140 & 3 & 24 \\
3 & $0.102(0.023)$ & 0.025 & 13 & 134 & 2 & 27 \\
Spring & $0.085(0.013)$ & 0.023 & 28 & 374 & 13 & 59 \\
1 & $0.075(0.023)$ & 0.023 & 8 & 127 & 0 & 18 \\
2 & $0.093(0.023)$ & 0.023 & 11 & 127 & 7 & 29 \\
3 & $0.087(0.020)$ & 0.024 & 9 & 120 & 6 & 25 \\
Summer & $0.100(0.015)$ & 0.027 & 30 & 367 & 5 & 66 \\
1 & $0.120(0.034)$ & 0.030 & 11 & 77 & 1 & 18 \\
2 & $0.100(0.024)$ & 0.023 & 7 & 131 & 3 & 26 \\
3 & $0.085(0.021)$ & 0.027 & 12 & 139 & & 27 \\
\hline
\end{tabular}

${ }^{1}$ Sample-to-positive.

tories, 2002). A logistic regression was performed conditioned on the previous response (i.e., positive or negative). This provided consideration for the probability that each subsequent positive or negative test result was conditional (i.e., not independent) on the previous result, while continuing to consider the effects of other explanatory variables.

Fecal culture results were described on the basis of both the proportion of cows exhibiting seropositivity and previous results of a positive or negative test (ELISA or fecal culture). The probability of a cow testing positive for MAP via fecal culture also was evaluated for the effects of mean daily maximum temperature in month of, month before, and 2 mo before sampling along with lactation number in a GLM framework (PROC GENMOD) using a binomial distribution and a logit link function. Using the repeated statement (by cow) and an auto-regressive (AR(1)) correlation structure, a GEE was utilized to adjust for the within-cow dependence of the outcome variable.

To assess potential biases affecting the long-term follow-up of the cohorts of cattle, the effect of each of seropositivity and $\mathrm{S} / \mathrm{P}$ ratio (categorized into quintiles) on the risk of culling was assessed using logistic regression (SPSS 12.0; SPSS Inc., Chicago, IL). In addition, survival analysis using Cox regression (SPSS 12.0) was used to assess within-lactation time to culling based on seropositivity and quintiles of S/P ratio. Both of the culling analyses were multivariable, adjusted for lactation number and DIM at the time of selection.

\section{RESULTS AND DISCUSSION}

The ELISA and fecal culture results are summarized in Table 2 for each season of sampling: fall, winter, spring, and summer, and by sampling cohort within season. For the subset (top $20 \%$ of ELISA S/P values at first test) of cattle with results from both fecal culture and ELISA in the same month, 234 of the samples exhibited both negative fecal culture and ELISA results, 30 samples had a negative fecal and a positive ELISA, 7 had positive fecal and a negative ELISA, and 21 were positive for both fecal culture and ELISA. Agreement between the 2 tests (ignoring within-cow dependence) was moderate (Kappa $=0.47 ; 95 \% \mathrm{CI}=$ 0.32 to 0.61 ). Overall, for 4 observed seasons, only 3 possible transition periods occurred (i.e., fall to winter, winter to spring, spring to summer). Of the 86 positive ELISA test results occurring during fall, winter, or spring, 18 had a negative test result sometime later during the study. Of the 947 negative results, 31 changed to a positive result. For all cows, sampling cohort; sampling season; sampling month; and mean, mean daily minimum, and mean daily maximum monthly temperatures in the month of, month before, and 2 mo before sampling did not affect $\mathrm{S} / \mathrm{P}$ ratio. Though non-significantly associated with the risk factors as listed previously, both negative-to-positive and positive-to-negative test results were observed in ELISA as well as fecal cultures for all 3 transitions. For fecal cultures, 155 cow-period transitions did not demonstrate a change in fecal culture test result; whereas 8 cow transitions from positive to negative and 8 cow transitions from negative to positive were observed. It is well documented that the sensitivity of the culture test is low, ranging from 33 to $50 \%$ (Sockett et al., 1992; Whitlock et al., 2000); thus, these types of transitions should be expected.

In the present study (conducted from October 2002 to September 2003), no consistent across-cohort seasonal 
effects were detected for S/P ratios or proportion seropositive to MAP, which had been observed previously in the historical (and less valid) cross-sectional timeseries data collected in 2001. In the mixed model analysis of $\mathrm{S} / \mathrm{P}$ ratio, however, a change $(P<0.05)$ in $\mathrm{S} / \mathrm{P}$ from the beginning to the end of the study was detected. Although the season-cohort interaction was not significant, the increasing S/P ratios were likely due to the first cohort (1) in which a trend toward increasing $\mathrm{S} / \mathrm{P}$ ratio over time was shown throughout the course of the study (Table 2). This is consistent with reports (Collins, 1996; Holmes et al., 2004), which suggested that serological response increases over time with advancement of the disease associated with advancing age; thus, the changes noted in our study are likely due to the stage of disease rather than season, because neither of the other 2 cohorts exhibited this increase in S/P ratio during the course of the study. It is also possible, however, that this finding was consistent with a seasonal effect. The single cohort involved with the significant change was the cohort first sampled in October 2002. It is possible that low $\mathrm{S} / \mathrm{P}$ values in that month were due to an extended lagged effect of the previous season. The other 2 cohorts began in November and December, respectively. However, because the results were inconsistent for the other models, it cannot be concluded that season had a significant effect on $\mathrm{S} / \mathrm{P}$ ratio results.

In the evaluation of the transitional model, no significant effects were found associating change in serological status with temperature. Variation in seropositivity within repeated samples per cow was noted on several occasions; however, these were consistent with other findings (Hirst et al., 2002). In addition, we found no evidence to support a hypothesis linking seasonality to the risk of MAP fecal culture positivity or change in status.

Two possible reasons exist for our inability to reproduce the results seen historically on this farm. First, the summer depression in both $\mathrm{S} / \mathrm{P}$ ratio and the proportion of cows seropositive to JD observed previously might simply have been an artifact, and not truly associated with seasonality. The other possibility is that an actual historical association with S/P ratio and seasonality existed, but shortcomings in the present study proved sufficient to thwart proper assessment of the association. Discussion of each of these possibilities follows.

Artifact. The historical data, for which declines in both S/P ratio and risk of seropositivity were observed during months of elevated temperature (Figure 1), were evaluated as a cross-sectional study with a single blood sample collected from specified cows only once per lactation. In other words, an entirely different group of cows was sampled during each subsequent month. It is possi- ble, therefore, that the large decreases in S/P ratio observed in the summer months of 2001 were related only to the features (e.g., age, infection status) of the specific groups of cows sampled at those times, rather than to climatic conditions at that time. It is for this reason that the present investigation was designed as a cohort study with repeated sampling to evaluate this observed phenomenon more properly.

An additional problem with the historical data may relate to the manner in which the blood samples were analyzed. The historical samples were analyzed on a monthly basis with no measures in place to eliminate or reduce potential plate-to-plate or inter-operator variation. Extremely high values for $\mathrm{S} / \mathrm{P}$ ratio, some $>6.0$, were recorded for certain cows. This result alone might have accounted for the decrease in the following months when the samples were analyzed on different plates and possibly by different operators (no automated devices were used by the laboratory at that time). Again, it is for these reasons that methods suited to reducing inter-plate and inter-operator variability in the evaluation of blood samples were specified in the present study.

True association. The historical cross-sectional data were obtained from a cooperator herd approximately 1 yr before the beginning of the present study. Mean temperatures that cows were subjected to during 2001 to 2002 were somewhat greater than those during 2002 to 2003, but the absolute temperature difference was not extreme. Rather, the key difference was in relative humidity, which was greater in 2001 compared with 2002. Because of this humidity difference, little or no significant cooling-off period occurred during the night, and the daily temperature humidity index (THI) was usually much greater. According to some authors (Kelley, 1982; Johnson, 1987), if the nighttime temperature cools to an acceptable thermoneutral zone (e.g., THI $=72$; Johnson, 1987), effects of any heat stress incurred during the day can be alleviated. As is noted in Figure 2, THI for the area and time period of the previous study were significantly greater than those during the current study, reaching almost 80 during certain periods, and especially $>72$ during the summer months. It could not be determined whether the cattle in the present study experienced periods of climatic heat sufficient to cause immunological suppression. It seems unlikely that a strictly linear dose-response relation between heat stress and the MAP test status would exist. Rather, a threshold effect at a critical THI would instead be expected. Therefore, statistical analyses that are predicated on treating heat indices as continuous (as opposed to categorical) variables could be problematic. Another aspect that might have exaggerated the results from the previous study was the fact that results 


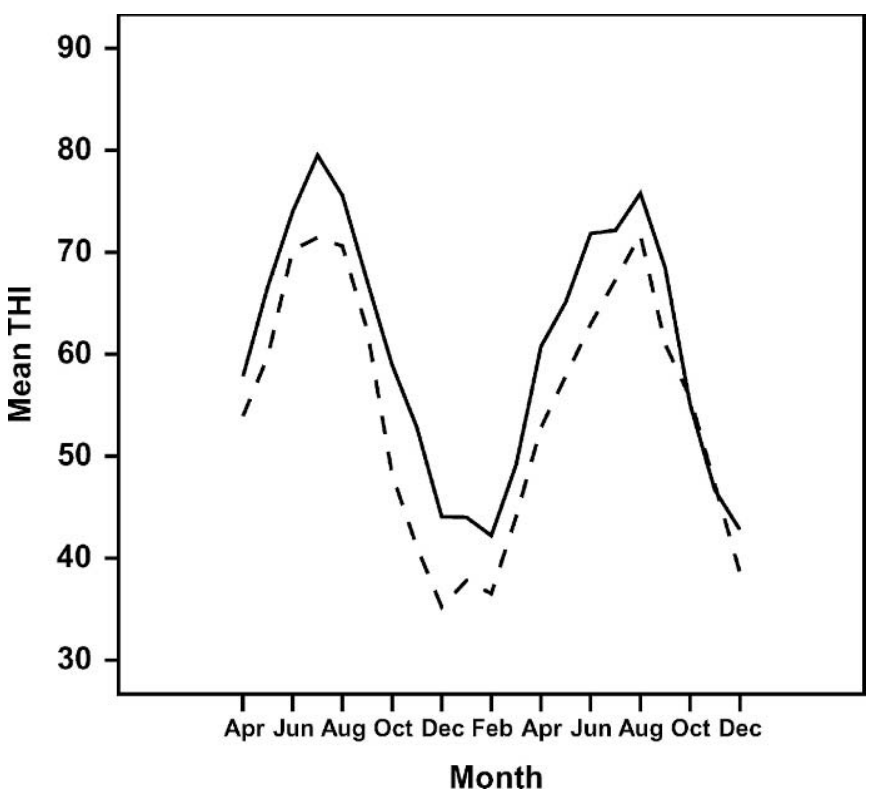

Figure 2. Mean monthly temperature humidity indices (THI) during the previous study (2001 to 2002; solid line) and during the current study (2002 to 2003; dashed line).

were obtained during a particularly hot summer, with temperatures greater than the normal average, even for that area (Figure 2).

A second issue that might have obscured any real relationship of S/P ratio with heat stress is that different groupings of cows were sampled in each of the respective studies. In the present study, one group of cows was followed over time; whereas in the previous study, different cows were sampled $1 \mathrm{mo} / \mathrm{yr}$, with eligibility for sampling established by their pregnancy status. Overall, mean S/P ratios for cows in the previous study were greater $(P<0.05)$ than those for the current study, with mean values of 0.158 for the historical study and 0.089 in the present study. Mean S/P ratios for the previous study were inflated by some of the extremely elevated observations in S/P ratio mentioned earlier, and so mean and median results (less susceptible to outliers) are shown in Figure 3. In addition, cows in the previous study had a mean monthly seropositive proportion of 0.099, whereas the present study had 0.067 seropositive. Prior to, and concurrent with the conduct of the historical cross-sectional study, whose data were collected in response to a managementdriven desire to manage colostrum from seropositive cows differently from seronegative animals, control measures for JD were implemented. Therefore, it is possible that, although the owner indicated he was not culling based on JD test results, some added culling pressure was applied to JD seropositive cows. In addition, the herd had expanded from approximately 2000

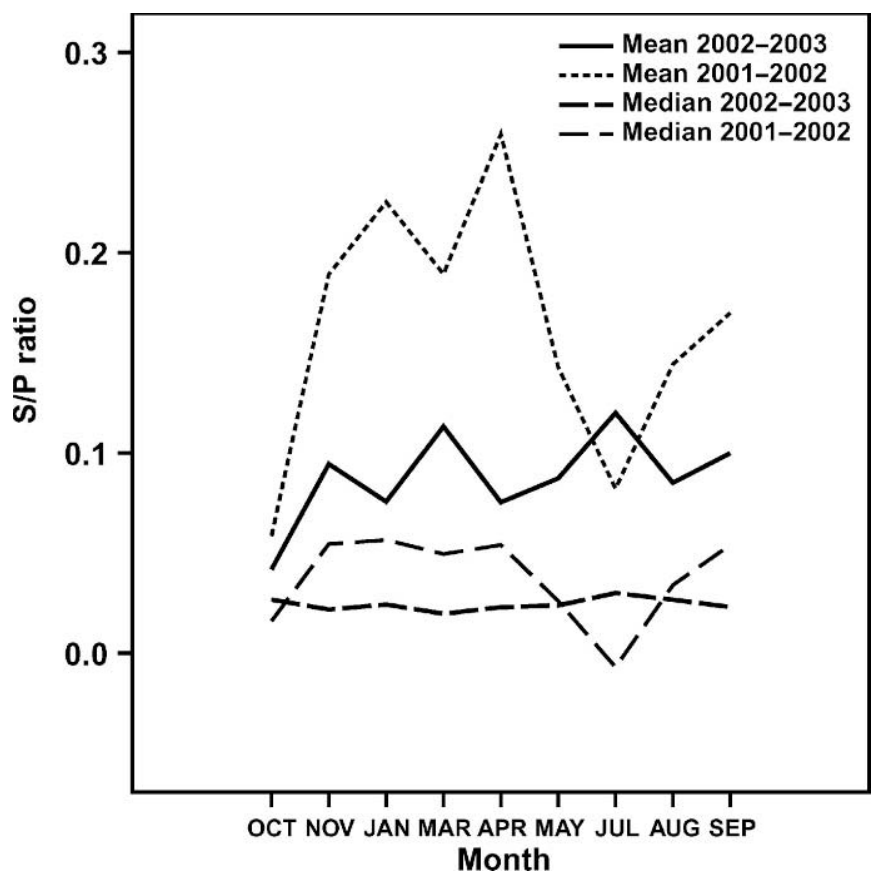

Figure 3. Mean and median sample-to-positive (S/P) ratios for jointly sampled calendar months during the previous sampling period (2001-2002) and the present study (2002-2003).

to 3400 cows from the time of the historical study to the current study; thus, unidentified stressors related to the expansion might have resulted in increased culling of JD positive cows.

A third issue that might have affected the results is the finding that Johne's positive cows were at a much greater risk for culling. In other words, a so-called healthy-worker survivor effect, common to occupational health studies of human workers (McMichael et al., 1976), might aptly have been applied in this longitudinal study design of dairy cows. This phenomenon in cohort studies of production livestock is well described (Dohoo et al., 2003). Because seropositive cows were being culled at a greater rate than seronegative cows, they were more likely to be lost to follow-up than seronegative or healthy cows. In the present study, the odds of a culled cow being positive for MAP infection by ELISA were approximately 5 times greater than that for cows that were retained in the herd, adjusted for lactation number. This was similar to results from another study (Wilson et al., 1993) in which a 6-fold increase in culling rate for seropositive cows was identified. In addition, cows with $\mathrm{S} / \mathrm{P}$ results in the top quintile (i.e., $>0.0819$ ) were culled from the herd at a faster rate (hazard) and were at a greater overall lactation risk $(32.1 \%)$ of culling when compared with those having smaller S/P ratios. For example, cows in the lowest S/P quintile $(<0.0083)$ were culled less rapidly 


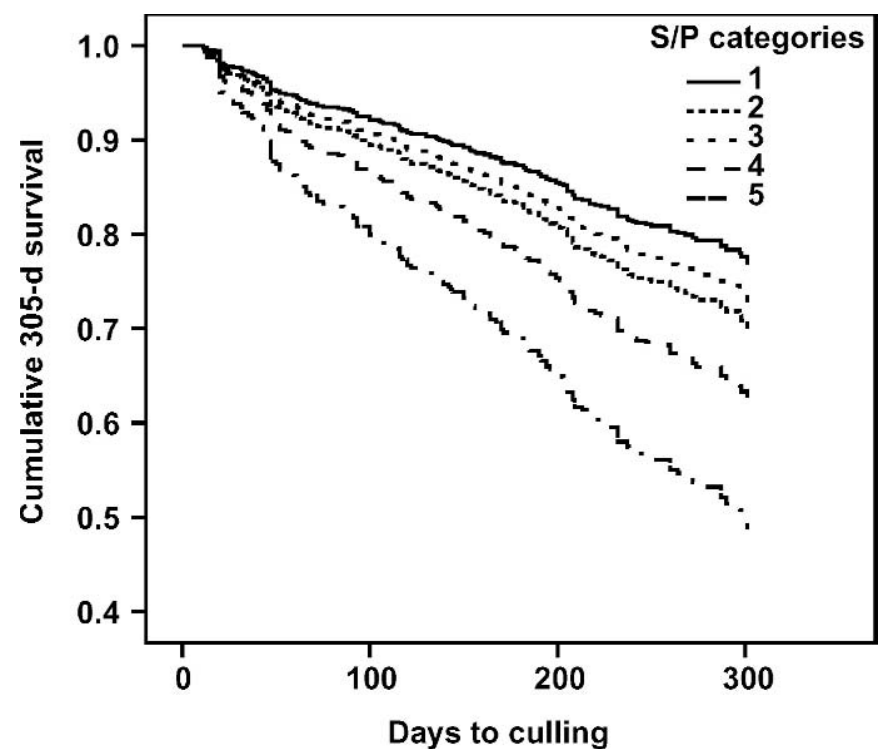

Figure 4. Survival plot of 305-d lactation time to culling by ELISA sample-to-positive $(\mathrm{S} / \mathrm{P})$ ratio quintiles consisting of the following categories: $1,<0.0083 ; 2,0.0083-0.0214 ; 3,0.0215-0.0395 ; 4,0.0396$ 0.0818 ; and $5, \geq 0.0819$.

(decreased hazard), with a lactation culling risk of 18.3\% (see 305-d survival curves in Figure 4, adjusted for lactation age). These culling risks were somewhat less than those identified by Goodell et al. (2000) in which cows with $\mathrm{S} / \mathrm{P}$ ratios $>0.10$ were removed from the herd at a rate of 35.8 to $50.0 \%$ compared with cows with an S/P ratio of $<0.09$ with a removal rate of $30.4 \%$. Because cows in our study with greater $\mathrm{S} / \mathrm{P}$ ratios were less likely to be present in the herd toward the end of the study, it might have been more difficult to describe any associations with season accurately. If elevated S/ $\mathrm{P}$ ratio results were missing from the already reduced mean $\mathrm{S} / \mathrm{P}$ ratios for the study population, it might have reduced the ability to discriminate significant fluctuations in $\mathrm{S} / \mathrm{P}$ ratio or proportion-infected data. It was imperative, in a seasonal study such as ours, to be able to follow the same cows over time (as best as is possible), and when a significant proportion of the cows were lost to follow up, seasonal variation was much more difficult to assess.

Because this 1-yr study began in the fall and ended in the summer, any subsequent increase in $\mathrm{S} / \mathrm{P}$ ratio or proportion of cows seropositive or fecal negative or positive to JD when colder months ensued could not be observed. If future studies were conducted, a springtime start date with a 2-yr study period would be ideal. The timeframe of funding for this study precluded a study period of more than a single year. In addition, the natural herd pressures of culling on seropositive animals (Figure 4) during a 2-yr period would make such studies difficult to undertake.

\section{CONCLUSIONS}

No significant seasonal effects on $\mathrm{S} / \mathrm{P}$ ratios or the proportion of dairy cows seropositive to MAP were observed. In addition, no evidence was found to support a hypothesis linking seasonality to an increased risk of MAP fecal culture positivity. At present, based on information derived from this study, the testing protocols currently being employed in JD control and management programs can be interpreted as being free of seasonal variability.

\section{ACKNOWLEDGMENTS}

This study was funded by USDA-APHIS grant \#029148-0717-GR. The efforts of the TVMDL laboratory scientists and staff and the cooperation and participation of the dairy producer and his staff are gratefully acknowledged.

\section{REFERENCES}

Collins, M. T. 1996. Diagnosis of paratuberculosis. Pages 357-371 in Paratuberculosis (Johne's Disease). Vet. Clin. North Am. Vol. 12. R. W. Sweeney, ed. W. B. Saunders Co., Philadelphia, PA.

Dargatz, D. A., B. A. Byrum, L. K. Barber, R. W. Sweeney, R. H. Whitlock, W. P. Shulaw, R. H. Jacobson, and J. R. Stabel. 2001. Evaluation of a commercial ELISA for diagnosis of paratuberculosis in cattle. JAVMA 218:1163-1166.

De Rensis, F., and R. J. Scaramuzzi. 2003. Heat stress and seasonal effects on reproduction in the dairy cow-A review. Theriogenology 60:1139-1151.

Diggle, P., P. J.Heagerty, K. Liang, and S. L. Zeger. 2002. Analysis of Longitudinal Data. Oxford University Press, New York, NY.

Dohoo, I., S. W. Martin, and H. Stryhn. 2003. Veterinary Epidemiologic Research. AVC Inc., Charlottetown, PEI, Canada.

Goodell, G. M., H. Hirst, F. Garry, and R. P. Dinsmore. 2000. Comparison of cull rates and milk production of clinically normal dairy cows grouped by ELISA Mycobacterium avium paratuberculosis serum antibody results. Proc. 9th Symp. Int. Soc. Vet. Epidemiol. Econ., Breckenridge, CO. Abstract ID 579. Coll. Vet. Med. Biomed. Sci., Colorado State Univ., Ft. Collins.

Hardin, J. W., and J. M. Hilbe. 2003. Cumulative logistic regression. Page 108 in Generalized Estimating Equations. Chapman \& Hall/ CRC, Boca Raton, FL.

Hirst, H. L., F. B. Garry, and M. D. Salman. 2002. Assessment of test results when using a commercial enzyme-linked immunosorbent assay for diagnosis of paratuberculosis in repeated samples collected from adult dairy cattle. JAVMA 220:1685-1689.

Holmes, I. R. L., T. F. Jubb, and A. P. L. Callinan. 2004. Infection rates in reactors to an absorbed ELISA used in a test and cull program for bovine Johne's disease. Aust. Vet. J. 82:233-235.

IDEXX Laboratories. 2002. Mycobacterium paratuberculosis Antibody Test Kit (Product Insert). IDEXX Laboratories Inc., Westbrook, ME.

Johnson, H. D. 1987. Bioclimate effects on growth, reproduction and milk production. Pages 35-57 in Bioclimatology and the Adaption of Livestock. Elsevier, Amsterdam, The Netherlands.

Kelley, K. W. 1982. Immunobiology of domestic animals as affected by hot and cold weather. Pages 470-479 in Proc. 2nd Int. Livest. Environ. Symp., Ames, IA. ASAE, St. Joseph, MI. 
Khare, S., T. A. Ficht, R. L. Santos, J. Romano, A. R. Ficht, S. Zhang, I. R. Grant, M. Libal, D. Hunter, and L. G. Adams. 2004. Rapid and sensitive detection of Mycobacterium avium subsp. paratuberculosis in bovine milk and feces by a combination of immunomagnetic bead separation-conventional PCR and real-time PCR. J. Clin. Microbiol. 42:1075-1081.

Lacetera, N., U. Bernabucci, B. Ronchi, D. Scalia, and A. Nardone. 2002. Moderate summer heat stress does not modify immunological parameters of Holstein dairy cows. Int. J. Biometeorol. 46:33-37.

McCullagh, P., and J. Nelder. 1989. Generalized Linear Models. Chapman and Hall/CRC, Boca Raton, FL.

McMichael, A. J. 1976. Standardized mortality ratios and the "healthy worker effect": Scratching beneath the surface. J. Occup. Med. 18:165-168.

NAHMS. 1997. Johne's Disease on U.S. Dairy Operations. USDA:APHIS:VS, CEAH, National Animal Health Monitoring System, Fort Collins, CO

Nielsen, S. S., C. Gronbaek, J. F. Agger, and H. Houe. 2002. Maximum-likelihood estimation of sensitivity and specificity of ELISAs and faecal culture for diagnosis of paratuberculosis. Prev. Vet. Med. 53:191-204.

SAS. 1996. SAS/STAT Software: Changes and Enhancements Through Release 6.12. SAS Inst., Inc., Cary, NC.
Sockett, D. C., D. J. Carr, and M. T. Collins. 1992. Evaluation of conventional and radiometric fecal culture and a commercial DNA probe for diagnosis of Mycobacterium-paratuberculosis infections in cattle. Can. J. Vet. Res. 56:148-153.

Stabel, J. 1997. An improved method for cultivation of Mycobacterium paratuberculosis from bovine fecal samples and comparison to three other methods. J. Vet. Diagn. Invest. 9: 375-380.

USAHA (United States Animal Health Association). 1998. U.S. voluntary Johne's disease herd status program for cattle. http:// www.aphis.usda.gov/vs/nahps/johnes/vjdhspusaha1.htm. Accessed Sep. 27, 2004.

USDA-APHIS. 2002. Uniform program standards for the voluntary bovine Johne's disease control program. http://www.aphis.usda.gov/vs/nahps/johnes/johnes-umr.pdf. Accessed Oct. 7, 2004.

Whitlock, R. H., S. J. Wells, R. W. Sweeney, and J. Van Tiem. 2000 ELISA and fecal culture for paratuberculosis (Johne's disease): Sensitivity and specificity of each method. Vet. Microbiol. 77:387-398.

Wilson, D. J., C. Rossiter, H. R. Han, and P. M. Sears. 1993. Association of Mycobacterium paratuberculosis infection with reduced mastitis, but with decreased milk- production and increased cull rate in clinically normal dairy-cows. Am. J. Vet. Res. 54:18511857.

Wolfenson, D., Z. Roth, and R. Meidan. 2000. Impaired reproduction in heat-stressed cattle: Basic and applied aspects. Anim. Reprod. Sci. 60-61:535-547. 